\title{
Ueber eine neue Art der Gattung Gnoriste $\mathrm{Mg}$.
}

Von V. v. Röder in Hoym (Herzogth. Anhalt).

(Ein dipterologischer Beitrag.)

Die Gatt. Gnoriste zählt bis jetzt 4 europäische beschriebene Arten. Schummel hat allerdings in der "Uebersicht der Arbeiten und Veränderungen der schles. Gesellsch. f. vaterl. Cultur", 1831, pag. 74, eine fünfte Art Gnoriste bivittata in litt. genannt, obne jedoch dieselbe näher zu beschreiben. Sie soll vom Harzgebirge stammen. Ich habe eine ähnliche Art im Harz gesammelt, die ich anfangs für Gnoriste bivittata Sclum. ansah; durch die Güte des Herrn A. Assmann, Assistenten am zoolog. Mus, in Breslau, wurde ich über die noch dort vorhandene Gnoriste bivittata Schum. aufgeklärt, so dass ich meine Art als neu erkenne. Ich nenne sie:

\section{Gnoriste Harcyniae n. spec.}

Nigro-fusca; fronte concolore; rostro palpisque nigris; antennis fuscis; thorace cinereo, vittis 3 nigro-fuscis, intermedia gemina; scutello maris fusco-nigro, feminae flavo; abdomine nigro-fusco; femoribus tibiisque testaceis; tarsis fuscis; alis cinereohyalinis. Long. $6-7 \mathrm{~mm}$. of $q$.

Ich sammelte diese Art Anfangs Mai an Felswänden des Hüttenberges im Selkethal an der Strasse nach Harzgerode.

Kopf und Fühler schwarzbraun; Stirn gleichfarbig; Rüssel viel kürzer als bei Gn. apicalis $\mathrm{Mg}$., nebst den Tastern schwarz. Thorax grau mit drei schwarzbraunen Striemen; die mittlere keulenförmig, hinten verküıt, durch eine feine graue Linie gespalten; die seitenständigen vorn verkürzt und den hinteren Rand erreichend. Am Seitenrand und auf den Interstitien der Längsstriemen befinden sich gelbliche Haare; das Schildchen ist beim of schwarzbraun, beim of gelb. Hinterleib schwarzbraun, fein weisslich behaart; die Haltzange des of roth bis schwarzbraun; die Lamellen der weiblichen Legröhre rothbraun. Hüften, Schenkel und Schienen gelb. Bei dem ô haben die Schenkel an der Basis meist einen schwarzen Punkt, manche q haben auch solche ohne diesen schwarzen Punkt; Tarsen gebräunt; die Sporen sind braun; der Metatarsus der vordersten Beine ist ungefähr so lang als die Schiene. Flïgel graulich glashell, die Randader geht etwas über die Spitze der dritten Längsader hinaus; kleine Querader länger als das

nWiener Entomologische Zeitung “ VI. Heft 4. (15. Mai 1887.) 
Basalstück der dritten Längsader; die Gabel der fünften Längsader unter der kleinen Querader beginnend. Schwinger gelb.

Die Art erinnert sehr an eine Boletina Staeg., weicht aber durch den verlängerten Rüssel von dieser Gatt. ab.

Die 4 anderen europäischen Arten von Gnoriste sind: Gnoriste apicalis Mg. Syst. Beschreib. I, p. 243, Tf. 9, Fig. 1-11. Gnoriste bilineata Zett. Dipt. Scand. XI, 4094, 2. Gnoriste trilineata Zett. Dipt. Scand. XI, 4095, 3. Gnoriste longirostris Siebke in Enum. Insect. Norveg. p. 244.

Ueber Gnoriste bivittata Schum in litt. gab mir H. Ass$\mathrm{m}$ a $\mathrm{n}$ folgende kurze Beschreibung: ${ }^{7} \mathrm{~mm}$ lang. Thorax, Beine und Legestachel lebhaft ockergelb, ersterer mit 2 ziemlich breiten schwarzen Striemen; Hinterleib gelblichgrau, die einzelnen Segmente gelb gerandet; Flügel graugelblich angehaucht. "

Die einzige exotische Gnoriste-Art, die ich kenne, ist Gnoriste megarrhina O. S. Western Diptera, p. 193, of ๆ. Yosemite Valley und von den White mountains (Nord-America) in meiner Sammlung, ausgezeichnet durch den sehr langen Rüssel, der beinahe eben so lang als der Körper und fadenförmig ist.

\section{I T E R A T U R.}

\section{Allgemeines.}

Hawer F. Annalen des $\mathrm{k}, \mathrm{k}$ Naturhistorischen Hofmuseums. Jahresbericht für 1886. (Wien 1887 bei A. Hölder; Separatabdr. aus B. II; 70 S.)

Wie der I. Band der gevannten Annalen enthält auch der II. Band in seinem 1. Hefte einen ausführlichen Jahresbericht über den Stand und über die Arbeiten des neuen k. k. Naturhist. Hofmuseums aus der bekannten Feder des umsichtigen und allverehrten Intendanten, des Herrn Dr. Fr. Ritter v. H a u er. Insbesonders werden die vaterländischen Zoologen den Herrn Berichterstatter über die Darstellung der Verhältnisse nnd Fortschritte des neuen Museums, dessen Erơffnung der Laie wie der Mann der Wissenschaft mit gleicher Sehnsucht erwarten, zu grossem Danke verpflichtet sein. Auch die Leser unserer Zeitung werden dem Jahresberichte für 1886 Interesse entgegenbringen, da er umständliche Aufzeichnungen über den Stand und über die Zuwächse der weltberühmten entomologischen Sammlangen des Museums enthält. (Preis des I. Heftes $1 \mathrm{fl}$.)

J. Mik.

\section{Dermoptera und Orthoptera.}

Krauss Herm. Die Dermopteren und Orthopteren Siciliens. (VerhandI. d. k. k. Zool.-Botan. Gesellsch, Wien 1837, pag. 1-22.)

Nach einer kurzen Einleitung, welche uns über die Verhältnisse der Orthopterenfauna Siciliens belehrt, und nach einer erschöpfenden Darstellun 


\section{$2 \mathrm{BHL}$ Biodiversity Heritage Library}

Röder, Victor von. 1887. "Ueber eine neue Art der Gattung Gnoriste MG." Wiener entomologische Zeitung 6, 155-156.

https://doi.org/10.5962/bhl.part.17746.

View This Item Online: $\underline{\text { https://www.biodiversitylibrary.org/item/44060 }}$

DOI: https://doi.org/10.5962/bhl.part.17746

Permalink: https://www.biodiversitylibrary.org/partpdf/17746

\section{Holding Institution}

Smithsonian Libraries

\section{Sponsored by}

Smithsonian

\section{Copyright \& Reuse}

Copyright Status: NOT_IN_COPYRIGHT

This document was created from content at the Biodiversity Heritage Library, the world's largest open access digital library for biodiversity literature and archives. Visit BHL at https://www.biodiversitylibrary.org. 